\title{
Exzellenz in der Bildung für eine innovative Schweiz: Die Position des Wirtschaftsdachverbandes Chemie Pharma Biotech
}

\author{
Marcel Sennhauser
}

\begin{abstract}
In Switzerland, the chemical, pharma and biotech industries make a substantial and sustained contribution to the Swiss economy. The company members of scienceindustries employ around 70,000 people in Switzerland. ${ }^{[1]}$ Since 1980 value creation and productivity have increased markedly. As a result the share of the Swiss gross value added has grown continually to reach 5.6\% in 2015. Exports have also increased. Today the chemical, pharma and biotech industry contributes around $45 \%$ of all Swiss exports and is therefore the largest Swiss export industry. ${ }^{[2]}$ This article describes the key requirements from the viewpoint of the chemical-pharma industry in order that Switzerland can continue to compete as an innovative location in global competition.
\end{abstract}

Keywords: Chemical, pharma, biotech industries · scienceindustries

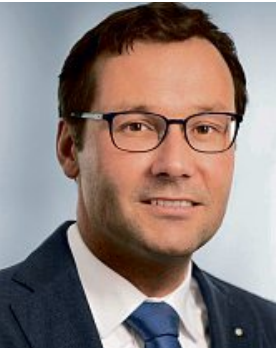

Marcel Sennhauser is Deputy Director General and Head of Communications of scienceindustries, the Swiss Business Association Chemistry Pharma Biotech in Zurich. He holds a higher polytechnic master degree HTL Lausanne in communication and an Executive MBA University of St. Gallen. He is Vice-Chairman of the SimplyScience Foundation, whose goal is to promote the motivation and understanding of adolescents for natural science and technology. Further he is Chairman of Swiss Engineering Media Ltd, which publishes various national journals for engineers and architects, and Vice-President of the Board of Tox Info Suisse, the Swiss national Toxicological Information Centre.

${ }^{*}$ Correspondence: M. Sennhauser

Mitglied der Geschäftsleitung

scienceindustries

Nordstrasse 15, Postfach

$\mathrm{CH}-8021$ Zurich

E-mail: marcel.sennhauser@scienceindustries.ch
Die Industrien Chemie Pharma Biotech leisten seit Jahrzehnten einen wichtigen und beständigen Beitrag zur schweizerischen Volkswirtschaft. Die Mitgliedsunternehmen von scienceindustries beschäftigen in der Schweiz direkt rund 70'000 Mitarbeitende. ${ }^{[1]}$ Die Wertschöpfung sowie die Arbeitsproduktivität konnten seit 1980 markant gesteigert werden. So erhöhte sich der Anteil an der Schweizer Bruttowertschöpfung kontinuierlich auf 5,6\% im Jahr 2015. Auch die Exporte sind Zug um Zug gestiegen. Heute erreicht Chemie Pharma Biotech einen Anteil von rund 45 Prozent aller schweizerischen Exporte und ist somit die grösste Schweizer Exportindustrie.[2] Der Beitrag beschreibt die zentralen Forderungen aus Sicht der chemischpharmazeutischen Industrie, damit die Schweiz weiterhin als innovativer Standort im globalen Wettbewerb bestehen kann.

\section{Innovationskraft als zentraler Pfeiler des wirtschaftlichen Erfolgs}

Der Erfolg dieser Industrie basiert auf ihrer im internationalen Vergleich herausragenden Innovationskraft. Dabei spielt die Spezialisierung auf wertschöpfungsintensive Produkte eine zentrale Rolle. Heute sind dies schwergewichtig die Herstellung und Vermarktung von Life-ScienceProdukten wie Pharmazeutika, Diagnostika, Vitaminen, Pflanzenbehandlungsmitteln sowie hochwertigen Fein- und Spezialitätenchemikalien.
Dazu sind bedeutende Investitionen in Forschung und Entwicklung notwendig. Von den 2015 insgesamt 22 Milliarden Franken, die in F\&E investiert wurden, werden rund zwei Drittel von der Privatwirtschaft ${ }^{[3]}$ geleistet. Der Anteil an öffentlichen Mitteln beträgt rund 24\% (Grafik 1), was im internationalen Vergleich unterdurchschnittlich ist.

Die Mitgliedsunternehmen von scienceindustries investierten dabei 2016 weltweit über 21 Milliarden Franken in F\&E. Davon gingen 32\%, was einem Gegenwert von 6,9 Milliarden Franken (Grafik 2) entspricht, in die Forschung und Entwicklung in der Schweiz. Damit ist Chemie Pharma Biotech die grösste Forschungsindustrie der Schweiz.

Um im internationalen Wettbewerb mithalten zu können, muss Chemie Pharma Biotech neben der finanziellen Investition auf hervorragend ausgebildete Arbeitskräfte bauen können. Als Voraussetzung ist dazu insbesondere eine erfolgreiche öffentliche Bildungs- und Forschungspolitik notwendig, die dem bestehende Mangel an Fachkräften entgegenwirkt und die zukünftige Nachfrage nach Hochqualifizierten sicherstellt.

\section{Chemie Pharma Biotech leistet in der Bildung einen wesentlichen Beitrag}

Die Mitgliedsunternehmen von scienceindustries tragen bei der Bildung ihren Anteil bei. So arbeitet der Verband in zahlreichen Forschungsprojekten der angewandten Forschung und der Grundlagen- 
Grafik 1. Finanzierung der F\&E nach Quellen. Quelle: BFS 2017.
Finanzierung der durchgeführten $\mathrm{F}+\mathrm{E}$ in der Schweiz nach Finanzierungsquelle, 2015

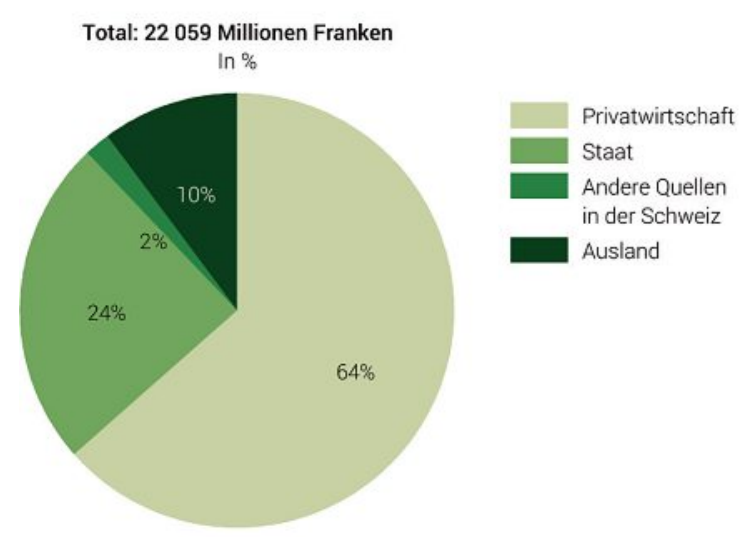

Quelle: BFS - Forschung und Entwicklung (F+E) Synthese Schweiz (FE Scheriz)

BFS 2017

\section{Forschungsausgaben der TopTen-Mitgliedsunternehmen von scienceindustries im In- und Ausland}

2009-2016, in Mrd. CHF; Total 21.5 Mrd. CHF im 2016

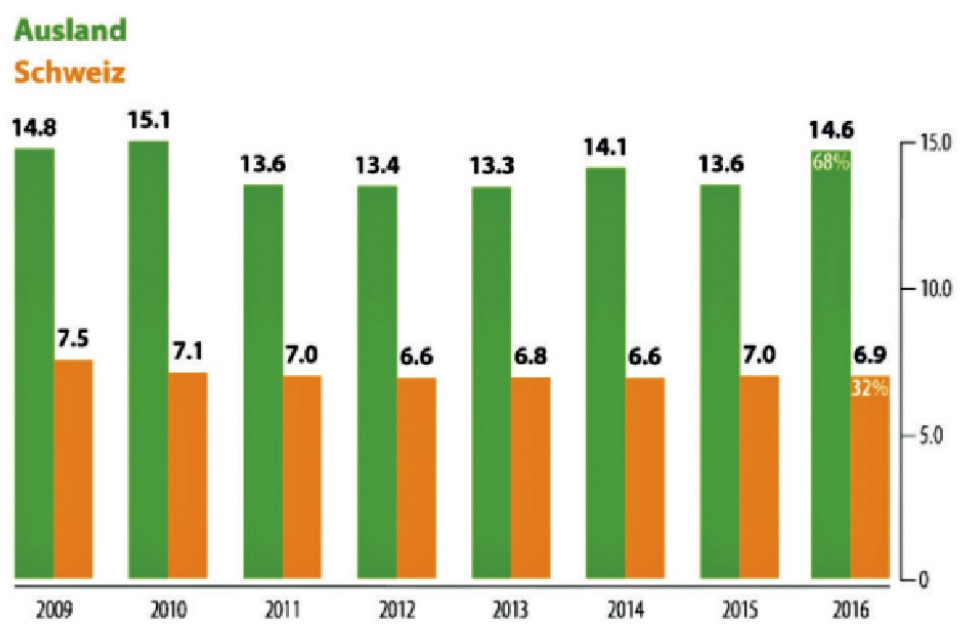

Grafik 2. Forschungsausgaben der TopTen-Mitgliedsunternehmen von scienceindustries im In- und Ausland, Quelle: scienceindustries 2017.

forschung mit den Schweizer Hochschulen und ausländischen Spezialisten zusammen. Weiter unterstützt er Schweizer Hochschulen mit finanziellen Mitteln zur Förderung begabter und leistungswilliger Nachwuchsforscher und des wissenschaftlichen Dialogs. Industrievertreter sind zudem als Dozenten und Referenten tätig und unterstützen Studierende bei ihren Diplomarbeiten. Zur Sicherung des Fachkräftenachwuchses bietet Chemie Pharma Biotech überdies rund 2000 Lehrstellen in naturwissenschaftlichen, technischen und kaufmännischen Berufen an und beschäftigt jährlich über 1000 Praktikanten, Doktoranden und Postdocs.

Die Industrie engagiert sich weiter mit verschiedenen Projekten und Initiativen für die Ausbildung des Nachwuchses. Dazu gehören insbesondere die Initiative SimplyScience ${ }^{[4]}$ zur Nachwuchsförderung in Naturwissenschaften und Technik (siehe separaten Artikel[5]) sowie Lehrmittel und Beiträge an ,,Schweizer Jugend forscht".

\section{Sicherung der exzellenten Bildung als Ziel der Schweiz}

Eine exzellente und zeitgemässe Ausbildung kann nur dann gewährleistet werden, wenn das gesamte Bildungssystem konsequent auf dieses Ziel ausgerichtet wird. Dazu braucht es den politischen Willen, Bildung überdurchschnittlich stark zu för- dern, gewissermassen Bildung als zentrales gesellschaftliches Fundament anzuerkennen. Die wichtigsten Elemente einer erfolgreichen Bildungspolitik werden in Graphik 3 dargestellt.

Aus Sicht von Chemie Pharma Biotech ist der Bildung und Forschung in allen öffentlichen Körperschaften gegenüber anderen Aufgaben strategische Bedeutung zuzumessen. Dabei sollen Bund, Kantone und Gemeinden für bildungs- und forschungsfreundliche Rahmenbedingungen sorgen und vermehrt auch konkrete Anreize für private Investitionen in die Bildung und Forschung schaffen. So sind beispielsweise fortschrittliche Stipendien- und Darlehenssysteme auf allen Schulstufen sowie bei einer Aus- oder Weiterbildung zu ermöglichen. Beim Steuerrecht sind die Steuerabzüge für Auszubildende, Arbeitnehmer, deren Arbeitgeber bzw. für Eltern mit Kindern in Ausbildung zu erhöhen, denn diese fördern die individuelle Bereitschaft zur Aus- und Weiterbildung. Dies kommt einer nachhaltigen Investition in das Humankapital gleich. Beim Stiftungsrecht ist das steuerliche Umfeld für Stiftungen zu verbessern.

\section{Leitprinzipien des Bildungssystems}

Bei der PISA-Erhebung 2015[6] liegt der Schweizer Mittelwert in den Naturwissenschaften über dem OECD-Durchschnitt. Der Mittelwert der Schweiz ist vergleichbar mit demjenigen von Deutschland, währenddem die anderen Nachbarländer (Österreich, Italien und Frankreich) deutlich tiefere Mittelwerte ausweisen. Deutlich an der Spitze finden sich die asiatischen Länder wie Singapur, Japan und China. Nur Finnland vermag als einziges europäisches Land bei der Spitzengruppe mitzuhalten.

Das Bildungssystem muss nach den Leitprinzipien Wettbewerb, Fokussierung auf das Wesentliche, Sicherstellung und Ausbau der Ausbildungsqualität sowie die Stärkung von naturwissenschaftlich-technischer Ausbildung im Allgemeinen sowie Chemie und Biologie im Speziellen ausgestaltet werden. Um die Verfügbarkeit von exzellenten Lehrkräften zu erhöhen und die gezielte Selektion zu ermöglichen, muss das öffentliche Ansehen und die Attraktivität des Lehrberufs verbessert werden. Denn gute Lehrer vermitteln nicht nur Inhalte, sondern sie wecken das Interesse und die Motivation der Schüler und stärken ihr Selbstvertrauen. Die didaktische und fachliche Aus- und Weiterbildung der Lehrerschaft in Chemie und Biologie soll weiter gestärkt werden. Dabei darf der Praxisbezug trotz der Akademisierung der Lehrerbildung nicht 
zu kurz kommen. Die Dozenten an den pädagogischen Hochschulen müssen über mehrjährige praktische Erfahrungen als Lehrpersonen verfügen. Die fachbezogene Weiterbildung der Lehrpersonen muss obligatorisch werden. Es ist zentral, dass sich die Lehrkräfte auf die Lehrtätigkeit konzentrieren können und nicht durch ständige Reformen und Zusatzbelastungen absorbiert werden.

Chemie Pharma Biotech unterstuitzt auf der obligatorischen Schulstufe (Primar und Sekundar I) die Programme Harmos und den Lehrplan 21. Die persönliche Beziehung zwischen Lehrperson und Schüler ist auf dieser Schulstufe für den Bildungserfolg besonders wichtig. Das Betreuungsverhältnis muss deshalb stetig verbessert werden. Die Integration in der obligatorischen Schule wird zwar gefördert, sollte aber auch ihre Grenzen haben. Eine $\mathrm{zu}$ starke Individualisierung des Unterrichts gefährdet die Qualität.

Das Interesse für Naturwissenschaften und dabei insbesondere für Chemie und Biologie sowie eine positive Einstellung zum technischen Fortschritt soll bereits im Kindergarten und in den ersten Schuljahren gefördert werden. Dabei sollen insbesondere die Mädchen gezielt gefördert werden.

Der Unterrichtskompetenz der Lehrkräfte ist besondere Aufmerksamkeit zu schenken, damit die Schweiz in Naturwissenschaften im Vergleich zur asiatischen Spitzengruppe konkurrenzfähig sein kann. Attraktive und zeitgemässe Lehrmittel sowie praxisbezogene Beispiele und Versuche helfen den Lehrpersonen, das Interesse und die Begeisterung der Kinder und Jugendlichen für Chemie und Biologie zu fördern. Für die Entwicklung der Lehrinhalte und Lehrmittel ist die $\mathrm{Zu}$ sammenarbeit mit der Wirtschaft zu verstärken.

Die Zulassungsbedingungen $\mathrm{zu}$ den Schulen der Sekundarstufe II (Maturitätsschulen, Berufsmaturitätsschulen sowie Wirtschafts- und Fachmittelschulen) sind messbar zu formulieren. Die Selektion soll der bestmöglichen Motivation und Entfaltung der Auszubildenden dienen. Durch falsche Selektion und in der Folge Unter- und Überforderung wird die Motivation untergraben und es werden Lebenswege versperrt.

Auch für die Maturität (Sekundarstufe II) sind landesweit verbindliche Bildungsstandards und vergleichbare Leistungsmessungen einzuführen. Die Matura muss eine ausreichende Qualifikation für ein Hochschulstudium in allen Disziplinen, so auch in Chemie und Biologie gewährleisten. Fixe Maturitätsquoten sind keine nachhaltige Lösung. Bei den Maturanden soll Quantität durch Motivation, Qualität durch Selektion sichergestellt werden.

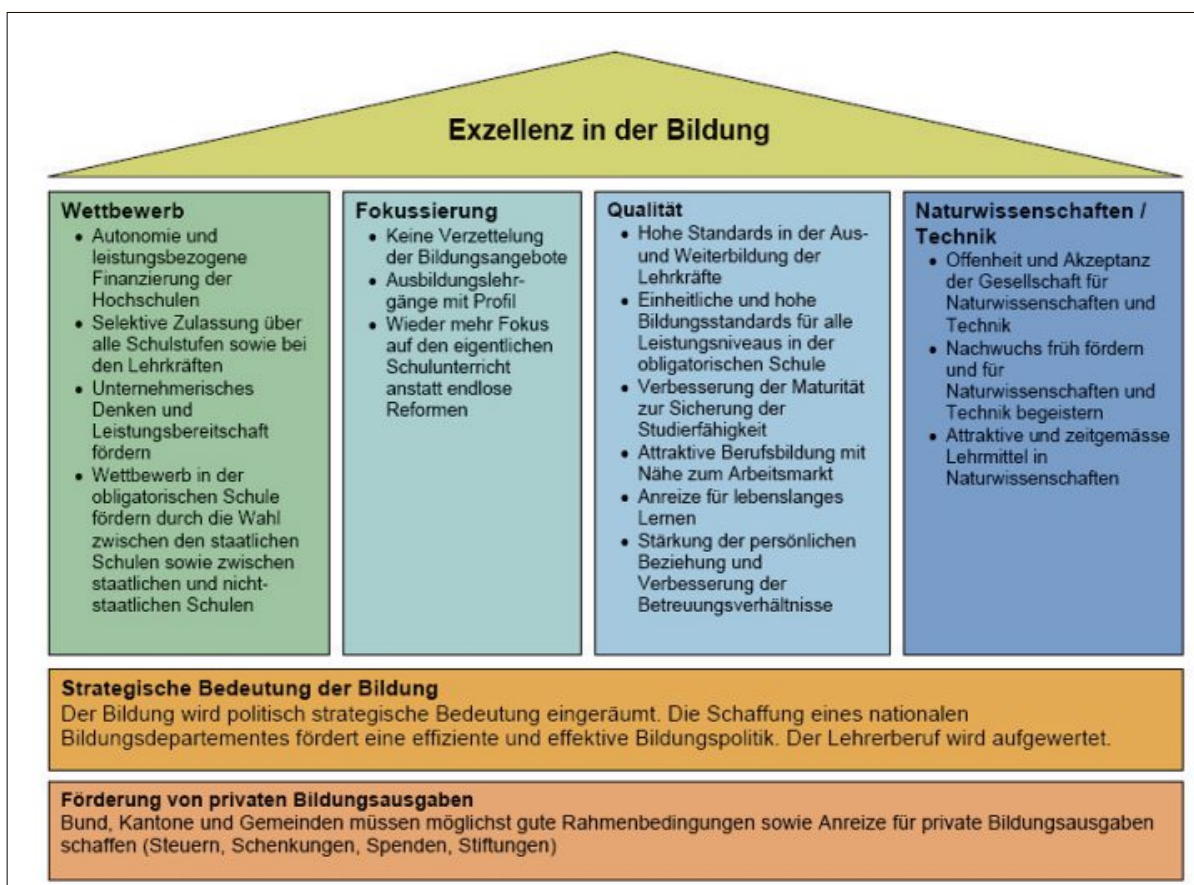

Grafik 3. Fundament und Pfeiler einer nachhaltigen Exzellenz in der Bildung (Quelle: scienceindustries 2013).

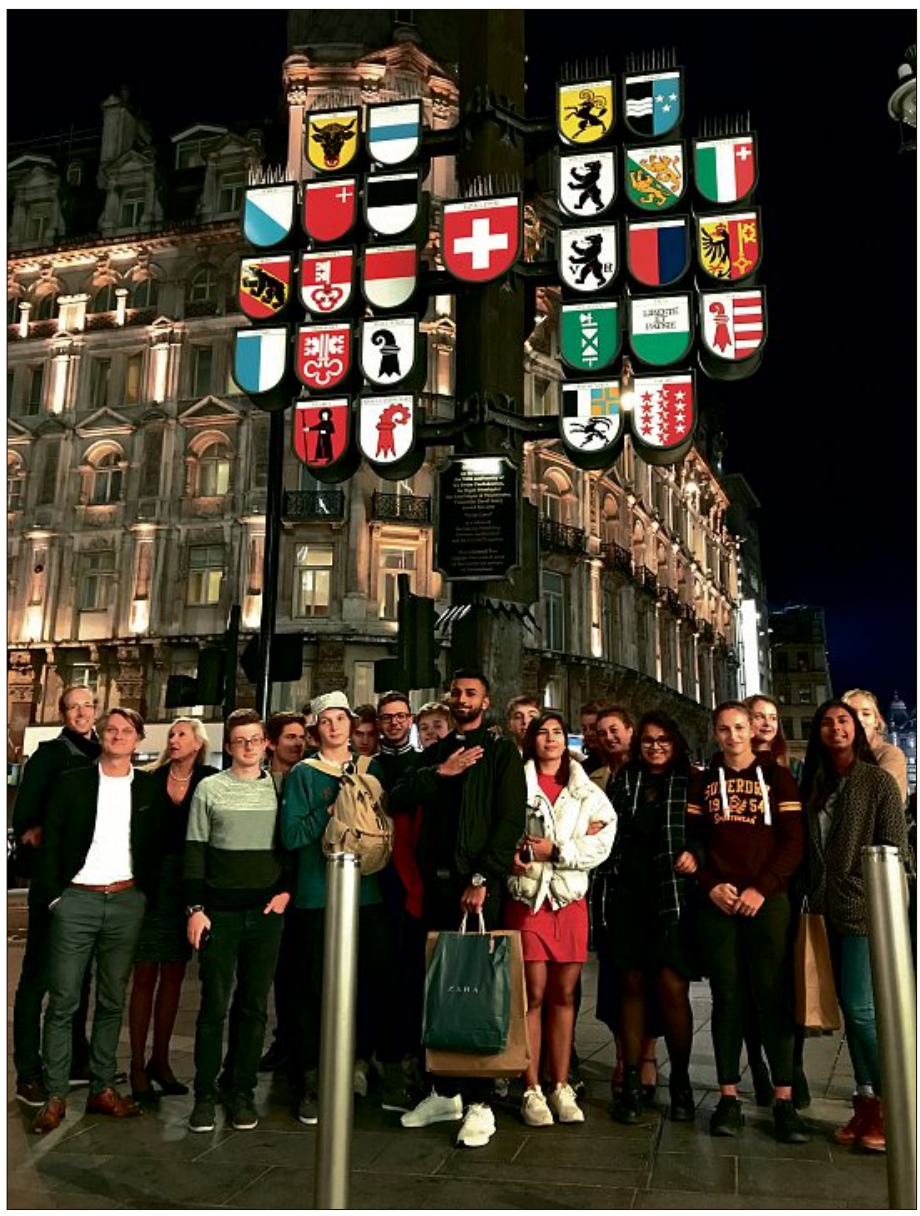

Figur 1. Die

Siegerklasse von "Science on the Move“, einem Programm von SimplyScience, in London.

Die Zulassungsbedingungen zur Tertiärstufe (ETH, Universitäten und Fachhochschulen) sind messbar zu formulieren. Insbesondere ist die heute während des ersten Studienjahres stattfindende Selektion weder individuell motivierend noch volkswirtschaftlich sinnvoll. Diese Selektion soll bereits in der Sekundarstufe II stattfinden.

Zwei Drittel der Schulabgänger wählen den beruflichen Ausbildungsweg. Die Berufsbildung muss ständig auf ihre Nähe 
zum Arbeitsmarkt und zum Qualifikationsbedarf der Wirtschaft überprüft und angepasst werden. Neue spezialisierte Qualifizierungen und Fachrichtungen sind frühzeitig in die Bildungsverordnungen $\mathrm{zu}$ integrieren.

Der Anteil der Berufsmaturandinnen und -maturanden muss weiter erhöht werden. Durch gezielte Information soll leistungswilligen Schülerinnen und Schülern aufgezeigt werden, dass der duale Bildungsweg mit Berufsmatur gute Bildungschancen und Karrieremöglichkeiten eröffnet.

\section{Mehr Qualität durch Wettbewerb und hohe Autonomie der Schulen}

Um international in der Ausbildung von Naturwissenschaften kompetitiv zu sein, ist der Wettbewerb zwischen den Schulen, gepaart mit Schulautonomie und Rechenschaftspflicht, hoch zu halten. Dies fördert unmittelbar die Qualität in der Schulbildung. Die freie Schulwahl ist zu etablieren. Privatschulen sind grundsätzlich nicht durch die öffentliche Hand zu finanzieren. Weiter soll das schweizerische Bildungssystem über alle Stufen sowie zwischen der beruflichen und der akademischen Bildung durchlässig sein.

Reformen sind unumgänglich, um die Exzellenz in der Bildung zu erreichen. Reformen müssen deshalb rasch und konsequent, aber nicht zu häufig durchgeführt werden. Reformen müssen die Chance erhalten, sich in der Praxis zu bewähren.

Schliesslich sollen Hochschulen (Tertiärstufe) im Rahmen von Globalbudgets und Leistungsverträgen selbstständig über ihr Bildungs- und Forschungsprofil sowie ihre Selektionskriterien entscheiden. Diese
Autonomie ist sicherzustellen. Die Mittelzuweisung an die Hochschulen soll nach Leistungskriterien erfolgen; der entsprechende Schlüssel soll nicht nur Studierendenzahlen, sondern auch Anzahl Diplome und Doktoranden sowie die Qualität von Lehre und Forschung berücksichtigen.

Der internationale Wettbewerbsdruck und die begrenzten finanziellen Mittel erfordern eine Angebotsbereinigung bei den Hochschulen. Nicht alle Hochschulen sollen in allen Leistungsbereichen Ausbildungen anbieten. Vielmehr soll über eine strategische Positionierung und den Leistungswettbewerb eine Differenzierung und Spezialisierung der Hochschulen erfolgen.

Die Fachhochschulen wiederum sollen im Vergleich mit universitären Hochschulen ein eigenständiges Profil aufweisen. Ihre Studiengänge sollen auf einer Berufslehre aufbauen. In der Regel soll der Bachelor der berufsbefähigende Abschluss sein, eine Ausnahme bilden die pädagogischen Hochschulen. Das Masterstudium an den Fachhochschulen soll sich ausschliesslich an herausragende Studierende richten. Doktoratsstudium und weiterführende Studien sollen den universitären Hochschulen und der ETH vorbehalten bleiben.

In der Schweiz treten die Hochschulabsolventen im Vergleich zu anderen Industrieländern spät in den Arbeitsmarkt ein. Die Ausbildungsgänge sollen deshalb ein klares Profil erhalten und zielgerichtet verlaufen, damit ein schnellerer Übertritt ins Berufsleben nach dem Studium möglich wird. Überdies sollen Anreize für einen raschen Abschluss des Studiums geschaffen werden.

Die Studiengebühren an den Hochschulen sind zu erhöhen, damit die Studie- renden sich bewusst für ein Studium entscheiden und sich die Studiendauer verkürzt. Dies ist insbesondere in den Naturwissenschaften vorzusehen, da diese Studienrichtung im Vergleich zu anderen eine kostenintensive Infrastruktur erfordert und daher aus volkswirtschaftlichen Gesamtüberlegungen den besten und interessiertesten Studenten zur Verfügung stehen sollen.

Dem akademischen Nachwuchs sind transparente und selektionsbetonte Karrierestrukturen anzubieten; dabei muss die Selektion internationalen Massstäben gerecht werden. Junge qualifizierte Talente und hochqualifizierte Lehrkräfte sollen unabhängig von ihrer Nationalität eingesetzt werden können. Es soll an den Hochschulen ein optimales Arbeitsumfeld geschaffen werden, um den drohenden 'brain drain' Hochqualifizierter ins Ausland zu verhindern.

Mit diesen zentralen Forderungen sieht die chemisch-pharmazeutische Industrie die Schweiz weiterhin als innovativen Standort im globalen Wettbewerb. Dies zu Gunsten der heutigen und zukünftigen Zivilgesellschaft und damit zur Sicherung des Wohlstandes aller in diesem Land.

Received: September 26, 2017

[1] https://www.scienceindustries.ch/industrie/beschaeftigungsstatistik

[2] https://www.ezv.admin.ch/ezv/de/home/themen/ schweizerische-aussenhandelsstatistik/publikationen/jahresberichte.html

[3] https://www.ezv.admin.ch/ezv/de/home/themen/ schweizerische-aussenhandelsstatistik/publikationen/jahresberichte.html

[4] www.simplyscience.ch

[5] T. Flüeler, Chimia 2018, 72, 11

[6] https://www.oecd.org/berlin/themen/pisa-studie/PISA_2015_Zusammenfassung.pdf 